\title{
Ethical Issues in AI-enabled Disease Surveillance: Perspectives from Global Health
}

\author{
Ann Borda ${ }^{1,5 *}$, Andreea Molnar ${ }^{2}$, Cristina Neesham ${ }^{3}$ and Patty Kostkova ${ }^{4}$
}

1 Centre for Digital Transformation of Health, University of Melbourne, Australia; aborda@unimelb.edu.au 2 Department of Computing Technologies, Swinburne University of Technology; amolnar@swin.edu.au

3 Newcastle University Business School, Newcastle University; cristina.neesham@ncl.ac.uk

4 Inst for Risk \& Disaster Reduction, University, University College London; p.kostkova@ucl.ac.uk

5 Department of Information Studies, University College London; a.borda@ucl.ac.uk

* Correspondence: aborda@ unimelb.edu.au

\begin{abstract}
Infectious diseases, as COVID-19 is proving, pose a global health threat in an interconnected world. In the last 20 years, resistant infectious diseases such as SARS, MERS, H1N1, Ebola, Zika and now COVID-19 have been impacting global health defences, and aggressively flourishing within the rise of global travel, urbanization, climate change and ecological degradation. In parallel, this extraordinary episode in global human health highlights the potential for artificial intelligence (AI)-enabled disease surveillance to collect and analyse vast amounts of unstructured and real-time data to inform epidemiological and public health emergency responses. The uses of AI in these dynamic environments are increasingly complex, challenging the potential for human autonomous decisions. In this context, our study of qualitative perspectives will consider a responsible AI framework to explore its potential application to disease surveillance in a global health context. Thus far, there is a gap in the literature in considering these multiple and interconnected levels of disease surveillance and emergency health management through the lens of a responsible AI framework.
\end{abstract}

Keywords: AI; disease surveillance; pandemics; global public health; ethics

\section{Introduction}

Infectious diseases such as COVID-19 pose a global health threat in an interconnected world. In the last 20 years, resistant infectious diseases such as SARS, MERS, H1NI, Ebola, Zika and now COVID-19 are impacting global health defences, and aggressively flourishing due to the rise of global travel, urbanization, climate change and ecological degradation.

In parallel, this episode in global human health highlights the potential for artificial intelligence (AI)-enabled disease surveillance to collect and analyse vast amounts of unstructured and real-time data to inform epidemiological responses. The exploration of the requirements of a global data-driven and epidemic intelligence surveillance system finds precedence in the literature [1] but the implementation has still a long way to go. A 2020 Lancet commentary provides insights into what might be possible in a collective approach to address the unpredictable and evolving COVID-19 transmission rates using AI [2]. But this promise is also hampered by the challenges of $\mathrm{AI}$, including the opaqueness of outputs derived from informal sources and the commensurate need for transparency at multi-stakeholder levels [3-4].

$\mathrm{AI}$ as a component of global health surveillance systems is relatively new and largely bounded by both specific applications and geographies [5-6]. Global health itself is built on national public health actions and institutions, primarily concerning population-wide interventions. However, global health is ideally concerned with all strategies for health improvement, whether population-wide or individually based health care actions. In other words, it goes across geographical administrative boundaries. There is no formalized definition for global health but Koplan et al [7] is widely cited for defining it as: "an 
area for study, research, and practice that places a priority on improving health and achieving health equity for all people worldwide".

Surveillance is a core function of both public health and global health practice. It is defined by the World Health Organization (WHO) as the "continuing scrutiny of all aspects of the occurrence and spread of disease that are pertinent to effective control". It is characterised by "methods distinguished by their practicability, uniformity, and frequently by their rapidity, rather than complete accuracy" [8].

The enabling components of surveillance systems may include laboratory diagnostics to detect or confirm health conditions; information technologies to support the surveillance processes of data collection, analysis, and dissemination; clinician consultation and reporting; public health education and training; legislation, regulations, and policies that support the conduct of surveillance and response. Communicable disease surveillance can operate at international, national, state, and local levels. Primary responsibility for public health action often lies with state and national health departments or public health agencies (e.g. Public Health England in the UK). For the purposes of this paper, WHO [9] has defined a disease epidemic as "the occurrence of cases of disease in excess of what would normally be expected in a defined community, geographical area or season". In practice, infectious disease data collection and analysis is complicated and encompasses a multi-stage process with several stakeholders across various organizational boundaries [10]. The main objective of infectious disease surveillance is to identify changes in incidence, either in the form of an acute outbreak (e.g. COVID-19) or a change in long-term trends [11].

Disease surveillance intelligence includes activities related to prompt identification of potential health hazards and their verification, assessment, and investigation to enable public health control recommendations [12]. The 2005 International Health Regulations (IHR) are designed to ensure timely recognition of outbreaks of infectious disease with the potential to spread widely [13]. The IHR 2005 also serves as a foundation for the Global Health Security (GHS) Agenda [14]. The GHS Agenda is "an effort by nations, international organizations, and civil society to accelerate progress toward a world safe and secure from infectious disease threats; to promote global health security as an international priority; and to spur progress toward full implementation of the IHR". They require WHO member nations to report outbreaks of international concern to the WHO within 24 hours of discovery [15].

For case-based surveillance, Member States can report using the WHO Outbreak Toolkit [16] to develop an initial case report. For example, consistent with the IHR, during the initial months of the pandemic (H1N1) in 2009, WHO requested that countries report the initial cases and thereafter the number of confirmed cases, and deaths throughout the H1N1 pandemic (declared 11 th June 2009). The resulting database represented one of the most comprehensive and timely outbreak reporting databases available to the public on the Internet at the time [13]. By contrast, the overwhelming human resource demands to effectively undertake COVID-19 data collection and analysis over the initial months of the pandemic swiftly required a shift from case-based reporting to aggregate and accelerated forms of reporting [17].

A critical component of disease surveillance which is growing in importance due to the availability of big data is 'epidemic intelligence'[18-19]. Epidemic intelligence incorporates two components: an indicator-based component and an event-based component $[9,12,20,21]$. The goal of indicator-based surveillance is to find increased numbers or clusters at a specific time, period, and/or geolocation that may indicate a threat [22]. The indicator-based component refers to structured or formal data collected through routine surveillance systems, such as the number or rates of cases based on standard case definitions, and the computation of indicators upon which abnormal disease patterns to investigate are detected [12]. Traditional indicator-based surveillance systems are based on the obligatory reporting of certain diagnosed diseases to a central health agency. 
Event-based disease surveillance systems use information on events impacting human health from Internet-based sources, including news aggregators and social media channels [21, 22, 23]. Event-based surveillance complements traditional approaches to public health surveillance and can provide early warning of emerging events, whereas there may be a lag in other forms of data aggregation due to delays in sample collection, laboratory confirmation, and country reporting, for example [24, 25]. The Medi+Board is an example of a dashboard framework which integrates public health surveillance data streams with zoonotic surveillance data [26] illustrating multiple geo-referenced and time series data.

There are several established and active disease surveillance systems utilized to monitor disease trends, mainly using online news sources, across the globe. Among these are: Global Public Health Intelligence Network (GPHIN), HealthMap, and ProMED [20, 21, 25, 27, 28].

Table 1. Disease Surveillance Systems

Global Public Health Intelligence Network (GPHIN) was established by the Public Health Agency of Canada in the late 1990s. GPHIN is an automated surveillance tool, which collects non-structured, event-based, digital data from news feed aggregators and then reports to national and international health agencies, such as the WHO Global Outbreak Alert and Response System (GOARN). In 2004, GPHIN detected severe acute respiratory syndrome (SARS) more than two months before the first publications by the WHO [29].

URL: https://gphin.canada.ca/cepr/aboutgphin-rmispenbref.jsp?language=en_CA

Healthmap, founded in 2006, uses online informal sources, such as ProMed and official-validated outbreak RSS feeds, for disease outbreak monitoring and real-time surveillance of emerging public health threats. HealthMap also brings together disparate data sources, including online news aggregators, eyewitness reports, expert-curated discussions and validated official reports. The freely available Web site 'healthmap.org' and mobile app 'Outbreaks Near Me' deliver real-time intelligence on a broad range of emerging infectious diseases.

URL: https://healthmap.org/en/

Program for Monitoring Emerging Diseases (ProMED) is a program of the International Society for Infectious Diseases (ISID). ProMED was launched in 1994 as an Internet service to identify unusual health events related to emerging and re-emerging infectious diseases and toxins affecting humans, animals and plants. ProMED is one of the largest publicly accessible surveillance systems conducting global reporting of infectious disease outbreaks.

URL: https://promedmail.org/

The IHR has emphasized the importance of both indicator-based and event-based components of epidemic intelligence for the early detection of events [30]. Informal information sources are important in this context, with the WHO reporting that more than $60 \%$ of initial disease epidemic reports come from unofficial sources and this percentage continues to increase with the availability of Internet-based and social media sources [25]. During the COVID-19 crisis, frequently changing government platforms being used to publicly disseminate data included Facebook and Twitter, as well as government authorized websites [31].

Common informal sources found on the Internet include search queries, news feeds (e.g. Google and Baidu news), blogs, and social media channels [25, 32]. Social media, such as Twitter, are perceived as an increasingly useful form of real-time data with a geolocation resolution that can be systematically mined, aggregated and analyzed by algorithmic applications to inform public health agencies [30, 33, 34]. The increasing number of 
smartphones worldwide and lower policy barriers in many lower-middle income countries (LMICs) also provide an opportunity for the usage of AI-enabled mobile applications in event-based surveillance [18, 35].

\section{AI and Public Health Disease Surveillance}

Rapid epidemic detection and real-time monitoring are critical objectives of eventbased surveillance to minimize the morbidity and mortality caused by infectious diseases and to enable preparation of rapid response public health services and personnel. Automated event-based surveillance systems have the potential capacity to aggregate and sort vast amounts of heterogeneous data for patterns and abnormalities using forms of AI [36].

AI machine learning classifiers play an important role in real-time analysis and trend predictions [37]. AI applications used with social media sources can be effective in providing trends for climatic and socio-economic contexts [38]. Social networks can further provide an efficient way for risk communication to prevent disease outbreaks and serve as real-time dissemination channels for declaration of pandemic events [39].

Machine learning models analyzing Internet and open health data sources has precedence across a number of disease surveillance projects to improve infectious disease surveillance and prediction e.g., malaria [40], dengue fever [41], and cholera [42]. In the example of influenza, algorithmic and machine learning models have been applied to influenza tracking for nearly two decades. Bernardo et al. [32] indicate that the use of Internetbased sources for disease surveillance is traceable to 2006, with early work focusing on influenza. Influenza can be highly contagious and easily spreads as people move about and travel, for instance, making tracking and forecasting flu activity a challenge. To address these challenges, researchers have proposed methods utilizing Internet-based sources, mobile phones, and other event-based surveillance methods. Research in the use of Internet data has demonstrated a positive predictive value of incidence of infectious diseases, e.g., seasonal influenza [43], but it has shown in some cases to have low predictive value [44].

To monitor the volume of influenza cases, there is an increasing use of "flu tracker" apps, whereby flu symptoms are reported using crowdsourced platforms to improve the global surveillance of influenza [35, 45]. The U.S. Centres for Disease Control and Prevention (CDC) developed the FluView app, which draws on the U.S. Influenza-like Illness Surveillance Network (ILINet) that records the percentage of patients reporting to outpatient clinics with symptoms of Influenza-Like Illness (ILI), such as fever, a sore throat or cough, over the total number of patient visits, for example. Data are integrated in the weekly joint WHO and ECDC COVID-19 and influenza (www.FluNewsEurope.org) bulletins [46].

These symptom measurements are an established indicator of historical ILI activity, but an important drawback is that they require several days to collect from individual health-care providers, causing delay and reducing the opportunity for real-time situational awareness and data analysis. In an evaluation of surveillance systems, it was found that the systems issued alerts for human cases between 1.9 and 6.1 days, on average, before WHO reports [30].

As monitoring is improved over time, the heuristics improve as their outputs are confirmed against a set threshold for the epidemiological attributes of extracted health events [22]. Prior to returning the extracted information, the surveillance system aggregates the extracted events into outbreaks, across several documents and sources based on this 'learning'.

The Computational Health Informatics Program (CHIP) at Boston Children's Hospital is among numerous research groups developing forecasting techniques combined with machine learning to provide more predictive indicators and estimates of local flu activity. An application called ARGONet (AutoRegression with General Online information) leverages information from electronic health records, flu-related Google searches and historical flu activity in a given location. In a research study, ARGO was shown to outperform 
Google Flu Trends (GFT) [47], a flu forecasting system developed by Google that operated from 2008 to 2015 but notably failed to accurately predict peaks in the flu season [25, 45, Daughton et al 2020]. To improve accuracy, ARGONet also draws on spatial-temporal patterns of flu spread in neighboring areas. "It exploits the fact that the presence of flu in nearby locations may increase the risk of experiencing a disease outbreak at a given location" [47].

In disease outbreaks before COVID-19, the application of AI was limited because of a shortage of data needed to provide rapid updates. The millions of feeds about coronavirus on social media and news sites are allowing algorithms to generate near-real-time information for public health services tracking its spread. For instance, the disease surveillance system HealthMap based at Boston Children's Hospital uses natural language processing and machine leaning to analyze data from government reports, social media, news site in 15 languages, and other informal sources to constantly track the spread of outbreaks [48]. Future developments at the time of this study include building a symptom-checker to assess the symptoms of coronavirus that distinguish it from seasonal flu.

In the emergence of COVID-19, risk analysis companies using AI-enabled tools were able to provide early detection of the coronavirus, before the WHO was informed. An AI epidemiologist in the Canadian-based company BlueDot (https://bluedot.global/) is widely reported as the first source to reveal news of the COVID-19 outbreak in late December 2019 [2]. BlueDot analyzed data from news reports, airline ticketing and animal disease outbreaks, to predict areas that would be prone to the outbreak, expanding from regions in China [48]. Data sources and AI played an important role in various countries' responses to the pandemic [18].

Another company using AI capabilities is Metabiota (https://www.metabiota.com/) based in San Francisco, which offers an Epidemic Tracker and a near-term forecasting model of disease spread. Metabiota, which also used analytics to track flight data accurately, anticipated that countries like Japan, Thailand, Taiwan and South Korea would be at risk of a coronavirus outbreak days before any case was reported in any of those countries $[48,49]$.

Interest in syndromic surveillance based on social media data has greatly increased in recent years, leading to what might be termed "Nowcasting" [47, 50, 51], that is providing predictions of disease levels which incorporate data from current social media activity but can include rolling data sources such as ever-hospitalized cases and persons tested [50]. The early detection and analysis of epidemics based on mining Twitter messages is a common approach in nowcasting [51-52]. The VAC Medi+Board dashboard provides visualizations of vaccination threads extracted from Twitter, for example, illustrating the diffusion of information, the most influential users, and impact groups [52-53].

Over the course of the swine flu (H1N1) pandemic in 2009, novel big data streams from social media channels, in correlation with traditional surveillance reporting, demonstrated a potential for early-warning systems for infection disease outbreaks detecting a peak in an outbreak of swine flu up to two weeks before the official public health authorities [54]. [34] reports that including Twitter data on influenza or influenza symptoms can improve nowcasting performance to a greater degree than was possible with search surveillance using Google Trends. During the 2012-2013 influenza season, a Twitter-based AI surveillance application also consistently predicted and mirrored CDC data with $85 \%$ accuracy [34].

COVID-19 big data applications are drawing on increasing aggregations of both formal and informal sources, including Twitter and major published open data platforms, e.g. Johns Hopkins University's COVID-19 global map dashboard. Pilot initiatives for COVID-19 forecasting, modelling and machine learning rose exponentially in 2020, demonstrating the capability of AI for disease surveillance applications [55]. The Global Partnership on Artificial Intelligence (GPAI) identified 84 initiatives supporting some form of AI tool, application and/or platform, from across the global north and south, related to the pandemic [56]. 


\section{Ethical Considerations}

There is a noted asymmetry in the literature on the ethics of AI in healthcare, with less attention granted to public health, particularly disease surveillance $[4,19,57,58]$. Much of the AI-driven intervention research in global health has limited description of ethical, regulatory, or practical considerations specifically required for cases of widespread use or deployment at scale [56,59].

Making AI systems transparent, fair, secure and inclusive is essential in this context, but how these systems are interpreted and operationalized can vary [6]. The increasingly promoted responsible AI frameworks have a potentially important application in disease surveillance, as touched on in the recent literature focusing on the COVID pandemic [56 $60,61]$.

However, this still remains a relatively unexplored area when applied to disease surveillance systems in global health. In terms of the components of Responsible AI, there are several frameworks that have been developed in the peer reviewed academic literature and in white papers [56, 58, 61, 70]. The Future Society in collaboration with the GPAI launched a responsible AI assessment framework aimed at applying a number of normative criteria to AI-related initiatives arising in the COVID-19 pandemic. This assessment is based on human rights, inclusion, diversity, innovation, and economic growth [56].

In an earlier and wider-ranging study, a meta-analysis of ethical AI frameworks by Floridi et al [62] resulted in the development of the AI4People framework for a 'Good AI Society', which is grounded in bioethics principles. The five principles underlying theAI4People framework are [62]:

- Beneficence: Promoting Well-Being, Preserving Dignity, and Sustaining

- the Planet

- Non-maleficence: Privacy, Security and "Capability Caution"

- Autonomy: The Power to Decide (Whether to Decide)

- Justice: Promoting Prosperity and Preserving Solidarity

- Explicability: Enabling the Other Principles Through Intelligibility and Accountability.

In applying the AI4People principles in the context of global public health disease surveillance, we further reflect on how such principles could inform both ethical and practical considerations for future disease surveillance system practices. We preface this discussion by noting that this conceptual approach inevitably presents limitations in the application of Responsible AI principles. Challenges that may not be covered by such principles, for example, point to finer grained considerations relating to the complexity of disease surveillance systems, from a system alert to reporting to different levels of health authority with varying governance capabilities and reliance on automated data generation and analysis in a global health emergency scenario.

\subsection{Beneficence: Promoting Well-Being, Preserving Dignity, and Sustaining the Planet}

This principle establishes the requirements for doing good, in the sense of pursuing the good pro-actively, as a constructive outcome, adding value to existing benefits. In this context, the role of a public health disease surveillance system, in itself, depends on a wider global health network, through which we can promote collaborative actions for the protection of people and the environment. Those systems of IHR Member States already have some form of embedded transparency in protecting and promoting the value of human dignity in the time of pandemics and in the process of disease surveillance. However, implementation of public health responses, particularly as post-surveillance events, as well as whole-of government approaches to public health disease outbreaks, need to also support and ensure a continuity of beneficence [70]. 


\subsection{Non-maleficence: Privacy, Security and "Capability Caution"}

This principle promotes the idea of avoiding harm, in particular to people, human society and the natural environment. In the context of this paper, key aspects of this 'do no harm' norm are data sharing, data tracking, data quality, and algorithm benchmarking.

\section{Data sharing:}

The quality and completeness of the data on which WHO relies depends on the capacity of its Member States for surveillance and their willingness to share surveillance data. As the COVID-19 pandemic progressed, Member States were asked by WHO to submit detailed case data via line lists on more than 80 variables, the vast majority of which were incompletely reported [17]. This recent pandemic, in particular, has highlighted significant challenges in sharing, storing and linking data from public, private, and quasipublic sectors, as well as between, across, and outside jurisdictions. Depending on the nature of the data, there may also be restrictions on linking or sharing outside of the geographical source of that data. In the context of new technology and innovation, it is becoming increasingly important for people to share their data, so that disease prevention advances can be more impactful. For example, the sharing of SARS-CoV-2 genetic sequences has allowed scientists to track bio evolution and variants [63].

\section{Data tracking:}

Here, there are concerns related to the privacy of the people whose data is being used, especially when data is linked from different sources such as self-reporting mobile apps or trackers, or CCTV video, making it easier to identify the people involved. Many countries relied on an extrapolation of infection-control and public-health measures to contain the COVID-19 pandemic. These have ranged from extreme quarantine measures in China to detailed contact tracing in South Korea to enforce stay-at-home policy and travel restrictions. Such measures have been variously implemented as COVID-19 contact tracing has revealed ethical tradeoffs between public health and privacy. For instance, in South Korea, there were human rights concern that the excessive disclosure of private information could cause people with symptoms to avoid testing [64].

Bluetooth privacy-presenting, anonymous, voluntary opt-in apps have been championed by human rights and data protection groups and supported by WHO [65]. The Decentralized Privacy-Preserving Proximity Tracing (DP-3T) protocol developed by several European academic institutions was among those developed in response to the COVID19 pandemic to safely facilitate digital contact tracing of infected individuals at the population level [66]. These were intended to offer a comprehensive set of technical and legal safeguards against potential misuse of personal data. However, a majority of individuals have not downloaded national digital contact tracing apps [66]. According to the Future Society report [56], public hesitancy querying the purpose of COVID-19 contact tracing apps has also emerged, caused by mistrust in public authorities and by fears around the establishment of continuing forms of government surveillance. Additional means of tracking and screening such as thermal image scanning are among the potential privacy breaches and creep of mass surveillance [67].

What should be the ethically acceptable trade-offs between stopping the spread of disease (a public good) and protecting a person's privacy from potential breaches (a private good)? This is a sensitive issue.

\section{Data quality:}

Real-world disease data tends to be noisy and incomplete. Although reporting of most notifiable diseases through various public health agencies is required by law, for the most part hospitals, laboratories and clinicians participate voluntarily. This reinforces the 
need for careful evaluation of data sources and collection procedures. Moreover, eventbased surveillance systems can generate a sizable amount of information on any given outbreak topic, sometimes overwhelming the systems themselves. For example, the Argus system generated approximately 22,000 reports on the pandemic (H1N1) 2009 from April 2009 to March 2010 [20]. In stark contrast, COVD-19 case reports captured early in the pandemic tallied 49,659 daily cases and 2,739 deaths for 27 March 2020 only [17]. Given the serious consequences of using poor quality data to manage global health related decisions, improving data quality becomes a crucial ethical concern.

\section{Algorithm benchmarking:}

Each syndromic surveillance system also implements a unique set of outbreak detection algorithms. This requires a better understanding of the strengths and limitations of various detection techniques and their applicability, which may require capability caution in the process [68]. For instance, algorithms can perform differently in the field than in the lab, and this may create a major challenge in ensuring high performance, accurate benchmarking against any current standard care, and continuously assessing performance after deployment. Given the wide and powerful impacts of decisions informed by such benchmarking, again, considerations of technical accuracy become ethical issues.

\subsection{Autonomy: The Power to Decide (Whether to Decide)}

The use of AI-based technology for global health surveillance also raises ethical questions around who should have the power to decide on these matters, and who should decide whether (and when) to decide at all. Concerns have been expressed about significant shifts of the locus of control on such decisions from humans to machines. In the context of global health surveillance, we note specificity, misinformation, consent and data governance as the main aspects that would require further ethical analysis (and, possibly, regulation).

\section{Specificity:}

In event-based surveillance, reports may be generated by automated machine-based processes, e.g. HealthMap, or by human analysts or subject matter experts,e.g. GPHIN, ProMED [25]. For the automated systems, manual report examination for relevancy typically occurs post-dissemination. ProMed utilizes local observers on the ground for some of its outbreak reporting; otherwise event-based biosurveillance systems often disseminate reports that are not observer or laboratory verified. Thus, although the reports provide near real-time alerts to users, the data they provide may not be specific enough. One consequence of this on human autonomy may be that individual human agents end up being constrained by limited one-size-fits-all (general) data and may not be able to see where (and how) they can act in different circumstances.

\section{Misinformation:}

Syndromic surveillance systems often generate false detections because it is sometimes difficult to distinguish natural data variations resulting from outlier and dynamic system changes, e.g. with time or space variables, from real outbreaks [69]. Human reviews and follow-up investigations are necessary for verification, which can be costly in time and labour. Scarcity of data, and lack of transparency in algorithms could compound challenges of misinformation or false alarms that could potentially occur with AI-enabled platforms, especially if they rely on social media or non-authoritative data sources, for example. False detections can erode public trust in these surveillance systems, and such misinformation could weaken an agency's or individual's power to effectively make decisions [70]. The role of social media in trending public attitudes can also be put to effective 
use to disseminate risk communication in real time [39] but could be a double-edged sword increasingly prone to misinformation and the spread of fake news [70,71].

\section{Consent:}

In an article by Blasimme and Vayena [57], the authors raised ethical questions around consent when employing AI-driven social media analysis for digital epidemiology. For example, ubiquitous surveillance for use by AI systems through personal devices, such as mobile phones, introduces the concern that granular data can be re-identified and personal health information can be hacked. The main concern here is that this personalized data can be used by third parties without the subject's knowledge or approval. In the case of contact tracing apps, for example, there are ethical considerations to gather and act on information without consent. Such digital surveillance on individual-level data can lead to unique complications [70] and relate to the trade-offs between public good and individual rights.

\section{Data governance:}

The COVID-19 pandemic has exposed long-standing data governance issues such as intellectual property rights, data sharing, reuse and ownership [56, 73]. Globally, persistent data gaps and fragmented approaches to governing disease surveillance data need to be addressed through global cooperation and clear, unified direction. The Global Pandemic Data Alliance (https://gpdahub.org/ ) is a collaboration of data-focused organizations across the G7 countries meeting the challenge of establishing health data as a global public good. This need for globally integrated data governance for public good is highlighted in the Future Society report [56] and in concurrent agency reports: WHO Health Data Governance Summit [74], UN Secretary General's Data Strategy, World Bank's 2021 Development Report, and 52nd UN Economic and Social Council Statistical Commission.

\subsection{Justice: Promoting Prosperity and Preserving Solidarity}

It is increasingly accepted that principles of justice should apply when AI is used to support event-based surveillance efforts in global health policy. Justice is not just a lofty ethical ideal, and an end in itself. It is also a means to distribute prosperity widely within society and to foster social cohesion and solidarity across different social groups. Geographic scope, human rights and equity in predictive decisions are the three main aspects of ethical concern to be discussed here.

\section{Geographic scope:}

The geographic scope of an event-based disease surveillance system may vary. It could cover a region, a country, a continent, or the entire globe. The following jurisdictions have established, hosted and maintained such systems: United States, European Union, Canada, Japan, Australia, Brazil, Singapore, Mexico, and Thailand. While there is immense potential opportunity for AI to support event-based surveillance of aggressive disease outbreaks such as COVID-19, the issue of missing data is especially true in low- and middle- income countries (LIC and LMIC), which may also lack infrastructure and human capital required to maintain these systems $[17,74]$. Geographic inequalities in event-based system maintenance may constitute significant barriers to achieving justice in global health outcomes, hence improvements in geographic coverage should become a priority.

\section{Human rights:}

Protection of human rights is paramount and, as stated in the UN Declaration of 1948, should be universally guaranteed. However, individuals from vulnerable groups and stateless communities may discover that current disease surveillance practices are still a long way from safeguarding them from discrimination and oppression. The IHR 
[15] has introduced important safeguards to protect the rights of travelers and other persons in relation to the treatment of personal data, informed consent, and non-discrimination in the application of health measures under the Regulations. The effectiveness of these measures on the ground remains to be determined.

\section{Predictive decisions:}

With respect to ensuring equity in predictive decisions, there is some debate as to whether responsible AI frameworks can address the explicit and implicit biases embedded within these systems [59]. To verify that an AI system is not using data in ways that result in bias or discriminatory outcomes, some level of transparency is necessary to explicitly addresses diversity and inclusion [56].

\subsection{Explicability: Enabling the Other Principles Through Intelligibility and Accountability}

It is now recognized that the 'black-box' nature of first-generation AI systems can be a source of injustice, dominance and harm $[4,68]$. The need for explainability (or explicability), in the sense of having algorithmic processes and outcomes explained in clear terms to the human intellect, has become evident. None of these processes and outcomes can be submitted to human judgment and scrutiny without certain explainability requirements being met. The main aspects we discuss here are: data noise; meeting regulatory standards or policy requirements; assessing risks, robustness and vulnerability; and understanding and verifying the outputs from a system.

\section{Data noise:}

Noisy data are a result of data corruption which can carry a large amount of additional or meaningless information. High noise data are a weakness of real-time data streams analysis which can introduce small errors that in turn can have an outsized effect on large-scale predictive models [75]. Some systems, such as HealthMap, relieve noise by integrating data from an assortment of online sources that have been moderated already [76].

\section{Meeting regulatory standards or policy requirements:}

Transparency is essential to legal compliance. Without it, enforcing individuals' legal rights in relation to the uses and applications of a technological system, establishing that a service meets regulatory standards, and determining liability may prove impossible [4]. While most nations have in place the procedures needed to foster compliance with existing data protection and privacy regulations, many such procedures are time consuming and create significant barriers to explicability $[17,38,74]$.

\section{Assessing risk, robustness and vulnerability:}

Understanding how a system works can be important in assessing risk [38]. This can be particularly important if a system is deployed in a new environment, where the user cannot be sure of its effectiveness. Interpretability can also help developers understand how a system might be vulnerable to so-called adversarial attacks, in which actors seeking to disrupt a system identify a small number of carefully chosen data points that they could alter in order to prompt an inaccurate output from the system [68].

\section{Understanding and verifying the outputs from a system:}

Interpretability can be useful in verifying the outputs from a system, by tracing how modelling choices, combined with the data used, affect the results. In some applications, this can help developers understand cause-and-effect relationships in their analysis. Furthermore, people are more likely to trust an algorithm when they can easily understand its implications and can modify it $[68,77]$. 


\section{Key issues of normative ethics}

$\mathrm{AI}$ is both a technical problem and a human problem, and scientific work on the application of AI in supporting disease surveillance must consider that it is ultimately humans who need to understand technology. From a normative-ethical perspective, we see two issues bearing on AI-based decision making in disease surveillance systems: (1) it is 'ethically blind' unless curated for value alignment (i.e. values shared by the human community are embedded in the algorithm); and (2) it complicates the issue of who is responsible for the consequences/outcomes of this decision-making process and their impact on various stakeholders.

The application of Responsible AI has considerable potential for supporting transparency which, in itself, promotes respect for the human dignity of stakeholders in the decision-making process. It also makes value alignment and judicious responsibility attribution possible in the first place. Without Responsible AI principles, meeting the two conditions systematically would be impossible. Further research should be engaged in developing effective approaches for value alignment in AI-assisted global health surveillance systems, as well as in normative-ethical analyses of responsibility attributions in the context of global health decision-making - the latter particularly in reference to the management of volatile pandemics, such as COVID-19.

\section{Conclusion}

AI models are increasingly used in decision-making contexts in global health disease surveillance. At the same time, they "are more complex and less interpretable than ever" [79]. Introducing Responsible AI applications could support a more ethical and equitable approach [62] to combatting pandemics and maintaining global public health [56]. However, the layered complexity and scale of global disease surveillance systems raise challenges for the integration of Responsible AI at different levels. These go beyond data, algorithm, prediction, and privacy concerns. The long-term goal relies on responsible health actors limiting the power of private corporate interests, ensuring transparency and protection of individual rights, and not least fostering trusted networks of partnerships and shared commitment through responsible global governance [80].

Author Contributions: The article was jointly written by all authors. All authors have read and agreed to the published version of the manuscript.

Funding: This research received no external funding.

Data Availability Statement: Not applicable.

Conflicts of Interest: The authors declare no conflict of interest.

\section{References}

1. Kostkova, P. A roadmap to integrated digital public health surveillance: the vision and the challenges. In Proceedings of the 22nd international conference on World Wide Web (pp. 687-694), 2013.

2. McCall, B.; COVID-19 and artificial intelligence: protecting health-care workers and curbing the spread. The Lancet Digital Health, 2020, Volume 2, No. 4, e166-e167.

3. Gordon, D.; Stavrakakis, I.; Gibson, J.P.; Tierney, B.; Becevel, A.; Curley, A.; Collins, M.; O'mahony, W.; O'sullivan, D. Perspectives on computing ethics: a multi-stakeholder analysis. Journal of Information, Communication and Ethics in Society. 2021

4. Morley, J.; Machado, C. C; Burr, C.; Cowls, J.; Joshi, I.; Taddeo, M.; Floridi, L. The ethics of AI in health care: a mapping review. Social Science \& Medicine, 260, 113172, 2020.

5. Khemasuwan, D.; Colt, H.G. Applications and challenges of AI-based algorithms in the COVID-19 pandemic. BMJ Innovations, 2021; Volume 7, No.2.

6. Piccialli, F.; di Cola, V. S.; Giampaolo, F.; Cuomo, S. The Role of Artificial Intelligence in Fighting the COVID-19 Pandemic. Information systems frontiers: a journal of research and innovation, 2021, pp. 1-31. Advance online publication. https://doi.org/10.1007/s10796-021-10131-x 
7. Koplan, J.P.; Bond, T.C., Merson, M.H., Reddy, K.S., Rodriguez, M.H., Sewankambo, N.K., et al. Towards a common definition of global health. Lancet. 2009;373:1993-5.

8. Last, J.M.; A dictionary of epidemiology. New York: Oxford University Press, 1988.

9. World Health Organization. Disease outbreaks. 2016. Available from: http://www.emro.who.int/health-topics/disease-outbreaks/index.html (Accessed on 12 July 2016)

10. Zeng, D.; Cao, Z.; Neill, D.B. Artificial intelligence-enabled public health surveillance-from local detection to global epidemic monitoring and control. Artificial Intelligence in Medicine. 2021, pp. 437-53. doi: 10.1016/B978-0-12-821259-2.00022-3. Epub 2020 Sep 11. PMCID: PMC7484813.

11. World Health Organization. Communicable disease surveillance and response systems: Guide to monitoring and evaluating. 2006. https://www.who.int/csr/resources/publications/surveillance/WHO_CDS_EPR_LYO_2006_2.pdf (Accessed on 8 November 2021)

12. Paquet, D.; Coulombier, R.; Kaiser,Ciotti, M. Epidemic intelligence: a new framework for strengthening disease surveillance in Europe. Euro Surveill. 2006, Volume 11, No. 12, pp. 212-214.

13. Baker, M.G.; Forsyth, A.M. The new International Health Regulations: a revolutionary change in global health security. $N Z$ Med J. 2007 Dec 14, Volume 120, No. 1267: U2872.

14. Global Security Agenda. https://ghsagenda.org (Accessed on 14 February, 2021)

15. World Health Organization. International Health Regulations. 2005. Geneva: http://whqlibdoc.who.int/publications/2008/9789241580410 eng.pdf. (Accessed on 21 May 2009)

16. World Health Organization. Outbreak Tookit. https://www.who.int/emergencies/outbreak-toolkit (Accessed on 14 February, 2021)

17. World Health Organization. Rapid Review of WHO COVID-19 Surveillance: External Review. 2021. https://www.who.int/publications/m/item/rapid-review-of-who-covid-19-surveillance-external-review-27-october-2021 (Accessed on 27 October 2021)

18. Kostkova, P.; Saigí-Rubió, F.; Eguia, H.; Borbolla, D.; Verschuuren, M.; Hamilton, C.; Azzopardi-Muscat, N.; Novillo-Ortiz, D. Data and Digital Solutions to Support Surveillance Strategies in the Context of the COVID-19 Pandemic. Frontiers in digital health. 2021, Volume 3, 707902. https://doi.org/10.3389/fdgth.2021.707902

19. Kostkova P. Disease surveillance data sharing for public health: the next ethical frontiers. Life sciences, society and policy. 2018, Volume 14, No. 1, pp. 16. https://doi.org/10.1186/s40504-018-0078-x

20. Nelson, N.P.; Yang, L.; Reilly, A.R. et al. Event-based internet biosurveillance: relation to epidemiological observation. Emerg Themes Epidemiol, 2012; Volume 9, No. 4. https://doi.org/10.1186/1742-7622-9-4

21. O'Shea, J. Digital disease detection: A systematic review of event-based internet biosurveillance systems. Int J Med Inform 2017; Volume 101, 15-22.

22. Velasco E, et al. Social Media and Internet-Based Data in Global Systems for Public Health Surveillance: A Systematic Review. Milbank Quarterly 2014; Volume 92, No. 1, 7-33.

23. Gajewski, K.N.; Peterson, A.E.; Chitale, R.A.; Pavlin, J.A.; Russell, K.L.; Chretien. J.-A. A review of evaluations of electronic event-based biosurveillance systems, PLoS One; 2014; Volume 9, No. 10

24. Chunara, R.; Freifeld, C. Brownstein J. New technologies for reporting real-time emergent infections. Parasitology 2012; Volume 139, No. 14, pp.1843-51.

25. Yan, S.J.; Chughtai, A.A.; Macintyre, C.R. Utility and potential of rapid epidemic intelligence from internet-based sources. International Journal of Infectious Disease. 2017, Volume 63, pp. 77-87. https://doi.org/10.1016/j.ijid.2017.07.020.

26. Kostkova, P.; Szomszor, M.; St. Louis, C. \#swineflu: The use of twitter as an early warning and risk communication tool in the 2009 swine flu pandemic. ACM Trans Manage Inf Syst. 2014, Volume 5, No. 2, Article 8 (July 2014). DOI: $10.1145 / 2597892$

27. Hartley, D.M., Nelson, N.P.; Walters, R.; Arthur, R.; Yangarber, R.; Madoff, L.; et al: The landscape of international event-based biosurveillance. Emerging Health Threats Journal. 2009, Volume 3

28. Walters, R.; Harlan, P., Nelson, N.P.; Hartley, D.M. Data sources for biosurveillance. Wiley handbook of science and technology for homeland security. Edited by: Voeller J. New York: John Wiley \& Sons; 2010; pp. 1-17.

29. Milinovich, G.J.; Williams, G.M.; Clements, A.C.A.; Hu, W. Internet-based surveillance systems for monitoring emerging infectious diseases, Lancet Infect. Dis. 2014, Volume 14, No. 2, pp. 160-168

30. Barboza P, et al. Evaluation of Epidemic Intelligence Systems Integrated in the Early Alerting and Reporting Project for the Detection of A/H5N1 Influenza Events. PLoS One. 2013; Volume, 8, No. 3; :e57252

31. Tsao, S. F.; Chen, H.; Tisseverasinghe, T.; Yang, Y.; Li, L.; Butt, Z. A. What social media told us in the time of COVID-19: a scoping review. The Lancet Digital Health, 2021; Volume, 3, No. 3; e175-e194.

32. Bernardo, T.M., et al. Scoping review on search queries and social media for disease surveillance: a chronology of innovation. J Med Internet Res 2013; Volume 15, No.7; :e147.

33. Guy, A.; Ratzki-Leewing, R.; Bahati, F.; Gwadry-Sridhar. Social media: a systematic review to understand the evidence and application ininfodemiology, Lect. Notes Inst. Comput. Sci. Soc. Inf. Telecommun. Eng. 2021; Volume 91, pp. 1-8, http://dx.doi.org/10.1007/978-3-642-29262-0 1. 
34. Broniatowski, D.A., Paul, M.J., Dredze, M. National and Local Influenza Surveillance through Twitter: An Analysis of the 20122013 Influenza Epidemic. PLoS ONE; 2013; Volume 8, No. 12; e83672. https://doi.org/10.1371/journal.pone.0083672

35. Mohanty, B.; Chughtai, A.; Rabhi, F. Use of Mobile Apps for epidemic surveillance and response - availability and gaps. Global Biosecurity; 2019; Volume 1, No. 2; pp.37-49. DOI: http://doi.org/10.31646/gbio.39

36. Davie, S. Artificial Intelligence in Global Health. Ethics E International Affairs; 2019; Volume 33, No. 2, pp. 181-192. https://doi.org/10.1017/S0892679419000157

37. Alessa, A.; Faezipour, M. A review of influenza detection and prediction through social networking sites. Theoretical biology $\mathcal{E}$ medical modelling, 2018, Volume 15, No. 1, 2. https://doi.org/10.1186/s12976-017-0074-5

38. Gluskin, R. T.; Mavinkurve, M.; Varma, J. K. Government leadership in addressing public health priorities: strides and delays in electronic laboratory reporting in the United States. American journal of public health, 2014; Volume 104, No. 3; e16-e21. https://doi.org/10.2105/AJPH.2013.301753

39. Szomszor, M.; Kostkova ,P.; St Louis, C. Twitter informatics: Tracking and understanding public reaction during the 2009 Swine Flu pandemic. IEEE Computer Society; 2011, pp. 320-323. Available: https://doi.org/10.1109/WI-IAT.2011.311

40. Ocampo, A. J.; Chunara, R.; Brownstein, J. S. Using search queries for malaria surveillance, Thailand. Malaria journal, 2013; Volume 12, 390. https://doi.org/10.1186/1475-2875-12-390

41. Hoyos, W.; Aguilar, J.; Toro, M. Dengue models based on machine learning techniques: A systematic literature review. Artificial intelligence in medicine, 2021, Volume 119, 102157. https://doi.org/10.1016/j.artmed.2021.102157

42. Abbasi J. Better Cholera Counts Through Machine Learning Models. JAMA. 2019, Volume 321, No. 14, pp. 1343. doi:10.1001/jama.2019.3459

43. Ginsberg, J.; Mohebbi, M. H.; Patel, R. S.; Brammer, L.; Smolinski, M. S.; Brilliant, L. Detecting influenza epidemics using search engine query data. Nature, 2009, Volume 457, No. 7232, pp. 1012-1014. https://doi.org/10.1038/nature07634

44. Daughton, A. R.; Chunara, R.; Paul, M. J. Comparison of Social Media, Syndromic Surveillance, and Microbiologic Acute Respiratory Infection Data: Observational Study. JMIR public health and surveillance, 2020, Volume 6, No. 2, e14986. https://doi.org/10.2196/14986

45. Smolinski, M.S., Crawley, A.W.; Baltrusaitis, K.; Chunara, R.; Olsen, J.M.; Wójcik O. et al. Flu Near You: crowdsourced symptom reporting spanning 2 influenza seasons. American journal of public health. 2015, Volume 105, No. 12, pp. $2124-30$. pmid:26270299

46. ECDC. COVID-19 surveillance guidance: Transition from COVID-19 emergency surveillance to routine surveillance of respiratory pathogens. ECDC Technical Report, October 2021.

47. Lu, F. S.; Hattab, M.W.; Clemente, C.L; Biggerstaff, M.; Santillana, M. Improved state-level influenza nowcasting in the United States leveraging Internet-based data and network approaches. Nature communications, 2019; Volume 10, No. 1; pp.147. https://doi.org/10.1038/s41467-018-08082-0

48. Allam, Z.; Dey, G.; Jones, D. Artificial Intelligence (AI) Provided Early Detection of the Coronavirus (COVID-19) in China and Will Influence Future Urban Health Policy Internationally. AI. 2020; Volume 1; pp. 156-165. doi:10.3390/ai1020009

49. Tong, S. Big Data Predicted the Coronavirus Outbreak and Where It Would Spread. https://www.marketplace.org/2020/02/04/big-data-predicted-coronavirus-outbreak-where-it-maygo-next/ (Accessed on 20 March 2020).

50. Greene, S.K.; McGough, S.F.; Culp, G.M. et al. Nowcasting for Real-Time COVID-19 Tracking in New York City: An Evaluation Using Reportable Disease Data From Early in the Pandemic. JMIR Public Health Surveill 2021; Volume 7, No. 1, pp. e25538 doi: $10.2196 / 25538$

51. Paul, M.J., Dredze, M., Broniatowski, D.A., \& Generous, N. (2015). Worldwide Influenza Surveillance through Twitter. In: Shaban-Nejad, A., Buckeridge, D.L., Brownstein, J.S. (eds.) Proceedings of the AAAI Workshop on the World Wide Web and Public Health Intelligence. 6-11.

52. Kostkova, P.; Mano, V.; Larson, H. J.; Schulz, W. S. Vac medi+ board: Analysing vaccine rumours in news and social media. In Proceedings of the 6th International Conference on Digital Health Conference. 2016; pp. 163-164.

53. Artus, D.; Larson, H.; Kostkova, P. Role of Social Media in vaccination debate about HPV: the VAC Medi plus Board study. European Journal of Public Health 2019. Oxford University Press.

54. Szomszor, M.; Kostkova, P.; Quincey, E. D. \# Swineflu: Twitter predicts swine flu outbreak in 2009. In International conference on electronic healthcare. 2010; pp. 18-26. Springer, Berlin, Heidelberg.

55. da Silva, C. C.; de Lima, C. L.; da Silva, A. et al. Covid-19 Dynamic Monitoring and Real-Time Spatio-Temporal Forecasting. Frontiers in public health, 2021; Volume 9, 641253. https://doi.org/10.3389/fpubh.2021.641253

56. Future Society. Responsible AI and AI in Pandemic Response, with the Global Partnership on AI (GPAI). https://thefuturesociety.org/2020/12/17/report-release-with-the-global-partnership-on-ai/(Accessed on 20 March 2020).

57. Blasimme, A.; Vayena, E. The Ethics of AI in Biomedical Research, Patient Care and Public Health (April 9, 2019). Oxford Handbook of Ethics of Artificial Intelligence. 2019. http://dx.doi.org/10.2139/ssrn.3368756

58. Murphy, K.,; Di Ruggiero, E., Upshur, R. et al. Artificial intelligence for good health: a scoping review of the ethics literature. BMC Med Ethics. 2021 Feb 15; Volume 22, No. 1; 14. doi: 10.1186/s12910-021-00577-8. PMID: 33588803; PMCID: PMC7885243.

59. Schwalbe, N.; Wahl, B. Artificial intelligence and the future of global health. Lancet. 2020; Volume 395, No. 10236; pp. 15791586. doi:10.1016/S0140-6736(20)30226-9 
60. El-Haddadeh, R.; Fadlalla, A.; Hindi, N. M. Is There a Place for Responsible Artificial Intelligence in Pandemics? A Tale of Two Countries. Information systems frontiers : a journal of research and innovation. 2021; pp. 1-17. https://doi.org/10.1007/s10796-02110140-w

61. Fosso Wamba, S., Queiroz, M.M. Responsible Artificial Intelligence as a Secret Ingredient for Digital Health: Bibliometric Analysis, Insights, and Research Directions. Inf Syst Front.; 2021; Volume 15; pp. 1-16. doi: 10.1007/s10796-021-10142-8. Epub ahead of print. PMID: 34025210; PMCID: PMC8122192.

62. Floridi, L.; Cowls, J.; Beltrametti, M. et al. AI4People-An Ethical Framework for a Good AI Society: Opportunities, Risks, Principles, and Recommendations. Minds and machines, 2018; Volume 28, No. 4; pp. 689-707. https://doi.org/10.1007/s11023018-9482-5

63. Jacob, J.J. et al. Evolutionary Tracking of SARS-CoV-2 Genetic Variants Highlights an Intricate Balance of Stabilizing and Destabilizing Mutations. mBio, 2021; Volume 12, No. 4; 12:e01188-21. https://doi.org/10.1128/mBio.01188-21, https://journals.asm.org/doi/10.1128/mBio.01188-21

64. Zastrow, M. South Korea is reporting intimate details of COVID-19 cases: has it helped?, Nature, 2020. http://dx.doi.org/10.1038/d41586-020-00740-y

65. World Health Organization. Ethical considerations to guide the use of digital proximity tracking technologies for COVID-19 contact tracing. Interim guidance, 28 May 2020. https://www.who.int/publications/i/item/WHO-2019-nCoV-Ethics Contact tracing apps-2020.1 (Accessed on 14 February 2021)

66. Blasimme, A.; Ferretti, A.; Vayena, E. Digital Contact Tracing Against COVID-19 in Europe: Current Features and Ongoing Developments. Front. Digit. Health. 2021; Volume 3:660823. doi: 10.3389/fdgth.2021.660823

67. Pisa M. COVID-19, information problems, and digital surveillance. Center for Global Development. 2020. Available at: https://www. cgdev. org/blog/covid-19-information-problems-and-digital-surveillance (Accessed on 15 February 2021)

68. Holzinger, A. et al. Causability and explainability of artificial intelligence in medicine. WIREs Data Mining Knowl Discov. 2019, Volume 9, No. 4; pp. e1312.

69. Chen, H. et al. AI for Global Disease Surveillance. IEEE Intelligent Systems, Volume. 24, No. 6, pp. 66-82, Nov.-Dec. 2009.

70. Aiello, A. E.; Renson, A.; Zivich, P.N. Social Media- and Internet-Based Disease Surveillance for Public Health. Annual Review of Public Health, 2020; Volume 41, No, 1, pp. 101-118.

71. Kostkova, P.; Szomszor, M.; St. Louis, C. \#swineflu: The use of twitter as an early warning and risk communication tool in the 2009 swine flu pandemic. ACM Trans Manage Inf Syst. 2014; Volume 5, No. 2, Article 8 (July 2014). DOI: 10.1145/2597892

72. Loomba, S.; de Figueiredo, A.; Piatek, S.J. et al. Measuring the impact of COVID-19 vaccine misinformation on vaccination intent in the UK and USA. Nat Hum Behav, 2021; Volume 5, pp. 337-348. https://doi.org/10.1038/s41562-021-01056-1

73. Godinho, M.A.; Borda, A., Kostkova, P. et al. Knowledge co-creation in participatory policy and practice: Building community through data-driven direct democracy. Big Data \& Society. 2021. January-June: 1-6. DOI: 10.1177/20539517211019430

74. World Health Organization. Health Data Governance Summit. Meeting Report 30 June 2021. https://www.who.int/publications/m/item/health-data-governance-summit (Accessed on 15 February 2021)

75. Ienca, M., Vayena, E. On the responsible use of digital data to tackle the COVID-19 pandemic. Nat Med. 2020; Volume 26, pp. 463-464. https://doi.org/10.1038/s41591-020-0832-5

76. Chen, H. et al. AI for Global Disease Surveillance. IEEE Intelligent Systems, Nov.-Dec. 2009; Volume 24, No. 6; pp. 66-82.

77. Dietvorst, B. J.; Simmons, J. P.; Massey, CAlgorithm aversion: People erroneously avoid algorithms after seeing them err. Journal of Experimental Psychology: General, 2015; Volume 144, No. 1, pp. 114.

78. Biran, O.; Cotton, C. Explanation and justification in machine learning: A survey. In IJCAI-17 workshop on explainable AI (XAI), 2017, Volume 8, No. 1; pp. 8-13).

79. Roberts, S., Kostkova, P. (2021). Disease Surveillance, Digital Futures, and Data-Sharing in a World 'After' COVID-19. Global Policy. 10 November 2021. https://www.globalpolicyjournal.com/articles/science-and-technology/disease-surveillance-digitalfutures-and-data-sharing-world-after

80. Arrieta, A.B. et al. Explainable Artificial Intelligence (XAI): Concepts, taxonomies, opportunities and challenges toward responsible AI. Information Fusion, 2020, Volume 58, pp. 82-115. https://doi.org/10.1016/j.inffus.2019.12.012. 